\title{
Direct Photon Production in Association With A Heavy Quark At Hadron Colliders
}

\author{
T. Stavreva and J.F. Owens \\ Department of Physics, \\ Florida State University, \\ Tallahassee, FL 32306-4350,USA
}

\begin{abstract}
Results of a next-to-leading order calculation of the inclusive cross section for a photon and a heavy quark (charm or bottom), $p \bar{p} / p p \rightarrow \gamma+Q+X$, are presented. Pointlike photon subprocesses through $\mathcal{O}\left(\alpha \alpha_{s}^{2}\right)$ and fragmentation subprocesses through $\mathcal{O}\left(\alpha_{s}^{3}\right)$ are included. The calculation is performed using a phase space slicing technique so that the effects of experimental cuts can be included. Results for the ratios of the charm and bottom cross sections are presented and the systematics of the various subprocesses for both the Tevatron and the LHC are compared and contrasted.
\end{abstract}




\section{INTRODUCTION}

Large momentum transfer processes have a long history of providing information on the substructure of hadrons, the nature of their constituents, and how they interact[1]. Photons provide an excellent probe for such purposes due to their pointlike electromagnetic coupling to the quark constituents of the colliding hadrons. The production of large momentum transfer photons has played dual roles of providing information of parton distribution functions (PDFs) [2] and testing the adequacy of the perturbative techniques used to calculate the hard scattering subprocesses [3], 4, $[5]$.

The single photon cross section, either inclusive or subject to photon isolation cuts, provides the basic observable for direct photon studies. The calculation of this cross section involves integrations over the phase space variables of the accompanying partons, thereby limiting the information which can be obtained about the underlying subprocesses. More information can be obtained if one can measure an associated jet as has been done recently by the DØcollaboration [6]. Even here, however, one is summing over many subprocesses with various flavors of partons. Additional information can be obtained if the flavor of the produced jets is tagged. Identifying jets which contain a heavy quark provides exactly this opportunity.

In this paper we investigate in detail one particular piece of the direct photon calculation, namely the associated production of direct photons and heavy quarks, where the heavy quarks are either charm or bottom. Some of the contributing subprocesses are dependent on the charge of the heavy quark while others are not. By considering both charm and bottom quarks one can examine the relative roles of the two contributions. In some kinematic regions the results are dependent on the heavy quark PDFs, opening the possibility of testing the current calculation of such PDFs.

New measurements of this process by the D $\varnothing$ and CDF groups are in progress and should be available in the near future. A comparison with these measurements will be presented in a forthcoming paper.

The production of heavy quarks at high- $p_{T}$ has the potential to generate logarithms of the form $\ln \left(p_{T} / m_{Q}\right)$ as a result of collinear configurations involving $Q \rightarrow Q g$ and $g \rightarrow Q \bar{Q}$. These logarithms can be resummed via the DGLAP equations for appropriately defined PDFs and fragmentation functions (FFs). This is commonly referred to as a variable flavor 
scheme with either four or five flavors. In such schemes the heavy quarks are treated as massless. The dominant remaining mass effect is due to the imposition of a threshold constraint such that the PDFs and FFs are taken to be zero when the hard scattering scales are smaller than the quark mass. The calculation presented here has been performed using the variable flavor scheme.

There have been previous studies of this process [7, 8, 9, 10]. In Ref. [7, 8] results are shown for the production of a direct photon plus charm. Here we also provide results for direct photon plus bottom production, and a comparison between the charm and bottom case. As noted above, this comparison depends on the relative roles of terms which depend on the heavy quark charge and those which do not. We also extend the calculation by treating the photon fragmentation contribution to next-to-leading-order (NLO). The previously cited references treated this component only in leading order (LO). One further technical point is related to the treatment of final state collinear singularities in the case when a gluon is emitted collinearly to a final state heavy quark. In Ref.[7] this singularity is factored into a charm FF. The present calculation is for the case of a photon produced in association with a jet which has been tagged as containing a heavy quark, so the fragmentation function is replaced by an appropriate jet definition.

In order to be able to work in the massless approximation the heavy quarks and photons produced need to carry a transverse momentum, $p_{T}$, which is few times larger than the mass of the heavy quark $m_{Q}$, i.e. $p_{T} \geq 10 \mathrm{GeV}$. Since the lower bounds for the values of the transverse momenta for direct photons and heavy quarks measurable at both the DØ and CDF collaborations at Fermilab are above $p_{T} \geq 10 \mathrm{GeV}$, a comparison with a massless calculation is appropriate. If however we are close to the threshold region for production of heavy quarks, i.e. $m_{Q} \sim p_{T}$, their mass needs to be retained, and they are assumed to be only produced externally in pairs, as end products of the hard-scattering. This is called the Fixed Flavor Number scheme (FFN), as the number of flavors that compose the nucleon remains fixed and it does not depend on the center of mass energy of the hard scattering. Here the proton is assumed to be composed only of light flavors, and in lowest order there are only two subprocesses in which the direct photon and heavy quarks can be produced. These are $g g \rightarrow \gamma Q \bar{Q}$, and $q \bar{q} \rightarrow \gamma Q \bar{Q}$. A study of the case when the quark masses are retained has been done at LO [9], where a comparison between the LO massive and massless approaches has been shown to give very similar results. In Ref. [9] a differential cross section in the 
transverse momentum of the photon up to values of $p_{T \gamma} \sim 50 \mathrm{GeV}$ is presented. There the difference between the two approaches in the LO is minimal.

This paper proceeds as follows: in Section II a description of the theory and techniques for the calculation are outlined. In Section III results for the differential cross sections are shown. Predictions for both the Tevatron and LHC are presented and compared. The effects of including the NLO fragmentation terms are shown, as well as the effect of the use of different charm PDFs on the cross section. In Section IV we summarize our findings.

\section{THEORY}
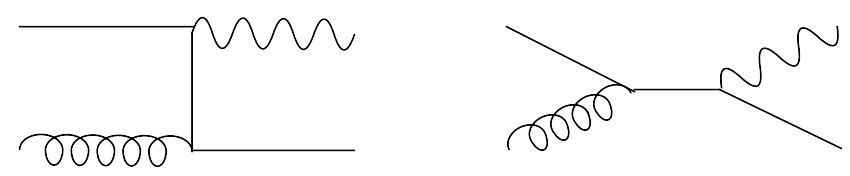

FIG. 1: Compton Scattering

Denoting the electromagnetic and strong couplings by $\alpha$ and $\alpha_{s}$, respectively, the lowest order subprocess for the production of a photon plus a heavy quark is the Compton subprocess, $g+Q \rightarrow \gamma+Q$, shown in Fig.1. This subprocess is of order $\alpha \alpha_{s}$ and in the variable flavor scheme employed here there is only this one subprocess to this order. When one considers higher order subprocesses such as $q Q \rightarrow q Q \gamma$, for example, there will be a region of phase space where the photon may be emitted collinear with the final state $q$, giving rise to a collinear singularity. This singular contribution may be absorbed into a photon fragmentation function $D_{\gamma / q}$. The photon fragmentation functions satisfy a set of inhomogeneous DGLAP equations, the solutions of which are of order $\alpha / \alpha_{s}$. More specifically, one has

$$
\begin{aligned}
& \frac{d D_{\gamma / q}(z, t)}{d t}=\frac{\alpha}{2 \pi} P_{\gamma / q}(z)+\frac{\alpha_{s}}{2 \pi}\left[D_{\gamma / q} \otimes P_{q q}+D_{\gamma / g} \otimes P_{g q}\right] \\
& \frac{d D_{\gamma / g}(z, t)}{d t}=\frac{\alpha_{s}}{2 \pi}\left[D_{\gamma / q} \otimes P_{q g}+D_{\gamma / g} \otimes P_{g g}\right]
\end{aligned}
$$

where $t=\ln \left(Q^{2} / \Lambda_{Q C D}^{2}\right)$ and $\otimes$ denotes a convolution. Writing $\alpha_{s}(t)=1 / b t$ it is easy to see that the solutions for both $D_{\gamma / q}$ and $D_{\gamma / g}$ are proportional to both $t$ and $\alpha$ so that the fragmentation functions may be thought of as being $\mathrm{O}\left(\alpha / \alpha_{s}\right)$. Therefore, another class of 


\begin{tabular}{|c|}
\hline NLO subprocesses \\
\hline$g g \rightarrow \gamma Q \bar{Q}$ \\
$g Q \rightarrow \gamma g Q$ \\
$Q q \rightarrow \gamma q Q$ \\
$Q \bar{q} \rightarrow \gamma q Q$ \\
$q \bar{q} \rightarrow \gamma Q \bar{Q}$ \\
$Q \bar{Q} \rightarrow \gamma Q \bar{Q}$ \\
$Q Q \rightarrow \gamma Q Q$ \\
\hline
\end{tabular}

TABLE I: a list of all $2 \rightarrow 3$ NLO hard-scattering subprocesses

contributions of order $\alpha \alpha_{s}$ consists of $2 \rightarrow 2$ QCD subprocesses with at least one heavy quark in the final state convoluted with the appropriate photon FF. An example is shown in Fig.2.

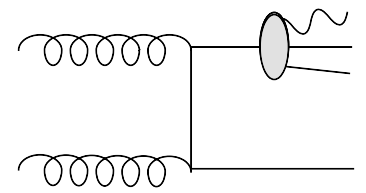

1)

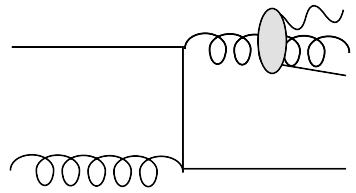

2)

FIG. 2: An example of Leading Order Fragmentation Contributions 1) $g g \rightarrow Q \bar{Q} \gamma$, where the photon can fragment off from either one of the final state heavy quarks, 2) $g Q \rightarrow g Q \gamma$, where again the photon can fragment off from either one of the final state partons, the gluon or the heavy quark

At the next order in perturbation theory, $\alpha \alpha_{s}^{2}$, the phase space for producing a photon in association with a heavy quark increases and now there are seven possible subprocesses, which are listed in Table 1. As in the LO case, there are fragmentation contributions that need to be taken into account in order to have a complete NLO calculation. Thus all $2 \rightarrow 3$ QCD subprocesses of order $\alpha_{s}^{3}$ once convoluted with $D_{\gamma / q, g}(z, Q)$, give something of the NLO: $O\left(\alpha_{s}^{3}\right) \otimes D_{\gamma / q, g} \sim \alpha_{s}^{3} \alpha / \alpha_{s}=\alpha \alpha_{s}^{2}$, Fig. 3 .

As soon as we go beyond LO, ultraviolet (UV), soft, and collinear divergences appear. The UV singularities arise from virtual diagrams when the momenta of the virtual particles go to infinity. To take care of these the theory is renormalized, and the singularities are 


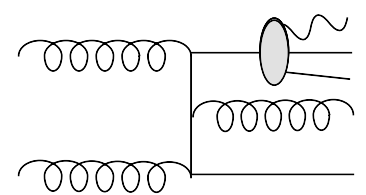

1)

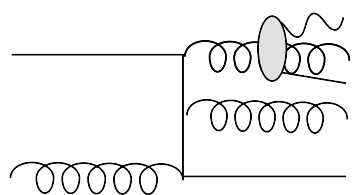

2)

FIG. 3: An example of Next-to-Leading Order Fragmentation Contributions 1) $g g \rightarrow Q \bar{Q} g \gamma$, where again the photon is produced by fragmenting from either of the final state partons produced in the hard scattering, 2) another example of NLO fragmentation $g Q \rightarrow g g Q \gamma$

absorbed into the now renormalized strong coupling $\alpha_{s}$. The soft singularities appear in the case where the energy of a massless particle like the gluon goes to zero, and the collinear singularities arise when two massless particles are emitted collinearly. Since generally the energies that are considered are much larger than those of the quark masses, the quarks are treated as massless, and the calculation is done in the massless approximation. To take care of the divergences the calculation is regularized. The regularization scheme that is used here is Dimensional Regularization (DR). In DR the scattering amplitudes are computed in $d=4-\epsilon$ dimensions, and the singularities are exposed as poles in $1 / \epsilon$. These poles cancel, once the virtual, soft and collinear contributions are added or they have to be absorbed into Parton Distribution Functions (PDFs) and FFs with the use of the DGLAP evolution equations. Once this is done it is safe to go back to 4 dimensions.

To perform the NLO calculation the two cutoff phase space slicing method [11] is used. In it the phase space is divided into $2 \rightarrow 2$ and $2 \rightarrow 3$ body contributions. The $2 \rightarrow 3$ body phase space is further divided into a hard region, where no singularities are present, a collinear region, where the collinear singularities are present and a soft region, where the soft singularities occur. The separation between the different regions is done with the help of two parameters : the soft cutoff $-\delta_{s}$, and the collinear cutoff $-\delta_{c}$. In the phase space slicing method a gluon is considered to be soft, if $E_{g}<\delta_{s} \sqrt{\hat{s}} / 2$, where $\sqrt{\hat{s}}$, is the hard scattering center of mass energy. In order to simplify the integration in this region the double pole or soft gluon approximation is used and the 4-momentum of the gluon is set to zero, when it appears in the numerator. The collinear region is taken to be where either $s_{i j}$ or $\left|t_{i j}\right|<\delta_{c} \hat{s}$, where $s_{i j}, t_{i j}$ are the Mandelstam variables. In the collinear region the leading pole approximation is used, so the relative transverse momentum of the two 
collinear particles in the numerators of the expansion is neglected. The integration over phase space is done with the use of Monte Carlo. This is very useful, as the cross section can be calculated as differential in any variable that is needed, such as the transverse momentum - $p_{T}$ or the rapidity - $y$, etc. of a given particle, without having to worry about calculating different Jacobians. There will be a dependence on the cutoff parameters in both the $2 \rightarrow 2$ contributions (which include the collinear and soft regions) and the $2 \rightarrow 3$ contributions, but this dependence will disappear once the two contributions are added together.

One final point needs to be addressed concerning the subprocess $q \bar{q} \rightarrow \gamma Q \bar{Q}$. There is a collinear singularity associated with the region where the final state $Q$ and $\bar{Q}$ are collinear. Physically, this corresponds to a $\gamma g$ final state with the gluon splitting into the $Q \bar{Q}$ pair. Normally, this singular region would be integrated over yielding a two-body contribution dependent on $\delta_{c}$ which would be proportional to the subprocess $q \bar{q} \rightarrow \gamma g$. This would be added to the one-loop corrections for the $q \bar{q} \rightarrow \gamma g$ subprocess, the poles in $\epsilon$ would cancel and there would be a residual $\delta_{c}$ contribution to the $q \bar{q} \rightarrow \gamma g$ subprocess. This would cancel against a similar contribution from $q \bar{q} \rightarrow \gamma Q \bar{Q}$ once a suitable jet definition was implemented in the calculation. However, once one tags the jet as containing a heavy quark, the problem arises in that there is no contribution from the subprocess $q \bar{q} \rightarrow \gamma g$. Hence, there is an uncanceled $\delta_{c}$ dependence in the $2 \rightarrow 3$ contribution. This problem is addressed by realizing that physically the final state gluon can not fragment into a $Q \bar{Q}$ pair unless its invariant mass exceeds $4 m_{Q}^{2}$. Imposing this constraint on the events generated for $q \bar{q} \rightarrow \gamma Q \bar{Q}$ avoids the problem of the uncanceled $\delta_{c}$ dependence.

\section{RESULTS}

\section{A. Tevatron Predictions}

For the numerical results shown below the CTEQ6.6M PDFs [12] were used, unless otherwise stated, with a 2-loop $\alpha_{s}$ corresponding to $\alpha_{s}\left(M_{Z}\right)=0.118$. The cross section was calculated for a center of mass energy of $\sqrt{S}=1.96 \mathrm{TeV}$ corresponding to the measurements being made at the Tevatron. The cuts applied reproduce the ones used by the DØ experiment, where the lower bounds for the transverse momenta of the photon and heavy quark are as follows: $p_{T \gamma}>30 \mathrm{GeV}, p_{T Q}>15 \mathrm{GeV}$, and their rapidities are limited to the central 


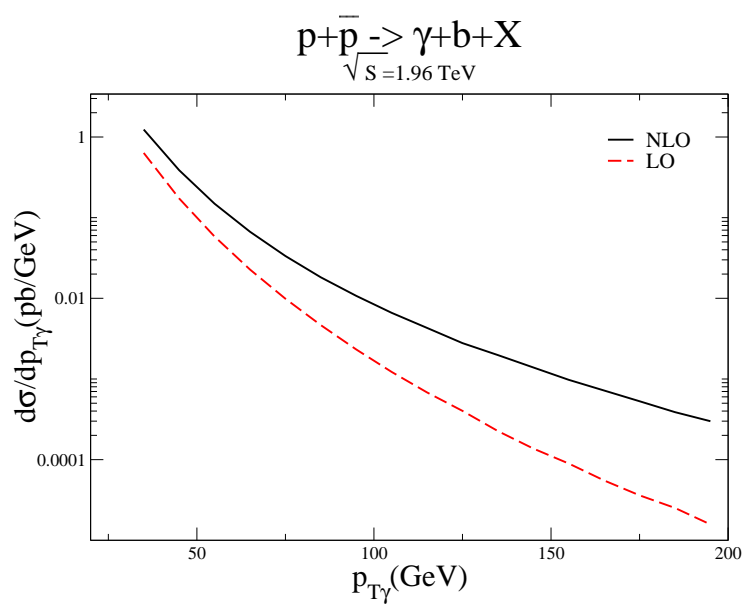

FIG. 4: The differential cross section, $d \sigma / d p_{T \gamma}$ for the production of a direct photon and a bottom quark as a function of $p_{T \gamma}$ for $\sqrt{S}=$ $1.96 \mathrm{TeV}$, at NLO - solid line, and at LO dashed line

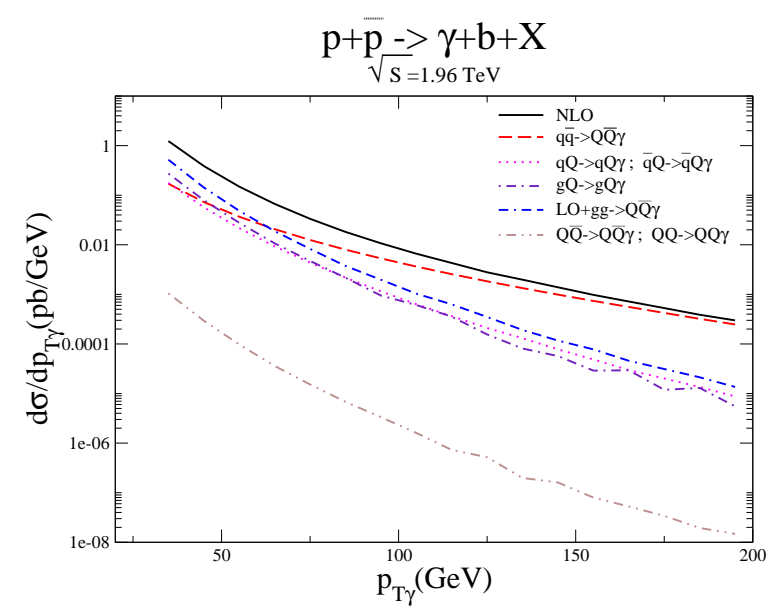

FIG. 5: contributions of the different subprocesses to the differential cross section, NLO solid line, annihilation $q \bar{q} \rightarrow Q \bar{Q} \gamma$ - dashed line, $q Q \rightarrow q Q \gamma$ - dotted line, $g Q \rightarrow g Q \gamma$ - dot dashed line, $g g \rightarrow Q \bar{Q} \gamma+\mathrm{LO}$ - dash dot dotted line, $Q \bar{Q} \rightarrow \gamma Q \bar{Q}$, and $Q Q \rightarrow \gamma Q Q$ - dot dash dotted line

region of the detector $\left|y_{\gamma}\right|<1,\left|y_{b}\right|<0.8$. If two final state partons lie within a cone of radius $\Delta R=0.5$, where $R=\sqrt{\Delta \eta^{2}+\Delta \phi^{2}}$ then they are merged into a single jet. If a final state has two heavy quark jets within the detectable region, it is counted only once, taking into account the transverse momentum of the more energetic jet. To be experimentally detectable, a photon needs to be isolated. This means that it should not be surrounded by hadronic energy more then $E_{h}=\epsilon * E_{\gamma}$ in a cone of radius $R=R_{\text {iso }}$ around it. The photon isolation requirements imposed model those needed in the D0 detection of a direct photon and are: $R_{1}<0.2, \epsilon_{1}<0.04$ and $R_{2}<0.4, \epsilon_{2}<0.07$.

The differential cross section for the process $p \bar{p} \rightarrow \gamma b X$ as a function of the transverse momentum of the photon is shown in Fig, 4. It is interesting to note in Fig 4 that as $p_{T \gamma}$ grows, the difference between the LO and NLO curves increases substantially. To understand the origin of this effect it is necessary to look at how the different subprocesses listed in Table 1 contribute to the cross section. This decomposition is shown in Fig.5.

It is apparent from Fig 5 that the effect shown in Fig 4 is driven by the annihilation 
subprocess, $q \bar{q} \rightarrow \gamma Q \bar{Q}$, which overtakes the Compton contribution and starts dominating the cross section at $p_{T \gamma} \sim 70 \mathrm{GeV}$. The $g Q \rightarrow \gamma g Q$ and $Q q \rightarrow \gamma q Q / Q \bar{q} \rightarrow \gamma q Q$ subprocesses contribute to the cross section about equally, with $g Q \rightarrow \gamma g Q$ prevailing over $Q q \rightarrow \gamma q Q$ $/ Q \bar{q} \rightarrow \gamma q Q$ at small $p_{T \gamma}$, where the gluon PDF is larger than the light quark PDF, and then at large $p_{T \gamma}, Q q \rightarrow \gamma q Q / Q \bar{q} \rightarrow \gamma q Q$ takes over when the light quark PDFs become larger than the gluon PDFs. The Compton subprocess and $g g \rightarrow \gamma Q \bar{Q}$ are added together, since the $g g \rightarrow \gamma Q \bar{Q}$ contribution is negative. This negative contribution is what remains after the appropriate collinear terms are subtracted. The size of the $2 \rightarrow 3 g g$ contribution is scale dependent, with the compensating collinear terms contributing to the $2 \rightarrow 2$ component. The role of the $Q \bar{Q} \rightarrow \gamma Q \bar{Q} / Q Q \rightarrow \gamma Q Q$ subprocesses is almost negligible, as the heavy quark PDFs are much smaller than the light quark and gluon PDFs.

Since the annihilation subprocess dominates the cross section at large $p_{T \gamma}$, it is useful to look at some of the Feynman diagrams contributing to it, as shown in Fig,6. There
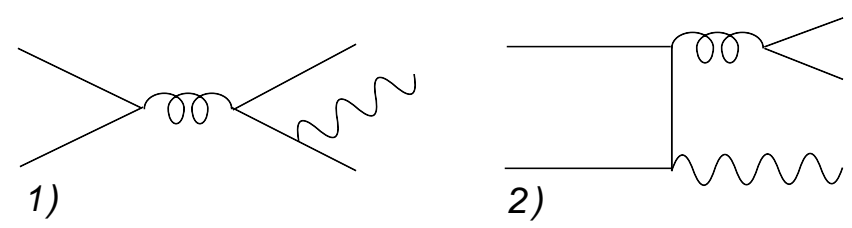

FIG. 6: Some typical Feynman diagrams for the annihilation subprocess $q \bar{q} \rightarrow \gamma Q \bar{Q}$ where 1) the photon is emitted from the final state heavy quarks and 2) the photon is emitted from the initial state light quarks

are two channels through which the annihilation subprocess can be produced, an s-channel shown in diagram 1) and a t-channel in diagram 2) of Fig, 6 . Since the photon couples to the final state heavy quarks in diagram 1), this diagram is proportional to the heavy quark charge, $e_{Q}$, whereas in diagram 2) the photon couples to the initial state light quarks, and thus this diagram is proportional to the light quark's charge, $e_{q}$. Diagram 2) begins to dominate as $p_{T \gamma}$ grows and, since it does not depend on the heavy quark charge, we expect the difference between the bottom and charm cross sections to decrease as $p_{T \gamma}$ increases. This indeed is the case as can be seen from Fig,7, where the NLO differential cross section for the charm quark (solid line) and the one for the bottom quark (dashed line) tend to come closer as the value of the transverse momentum increases. However, the difference between the LO cross sections stays about the same as can be seen from the dot-dashed (charm) and 


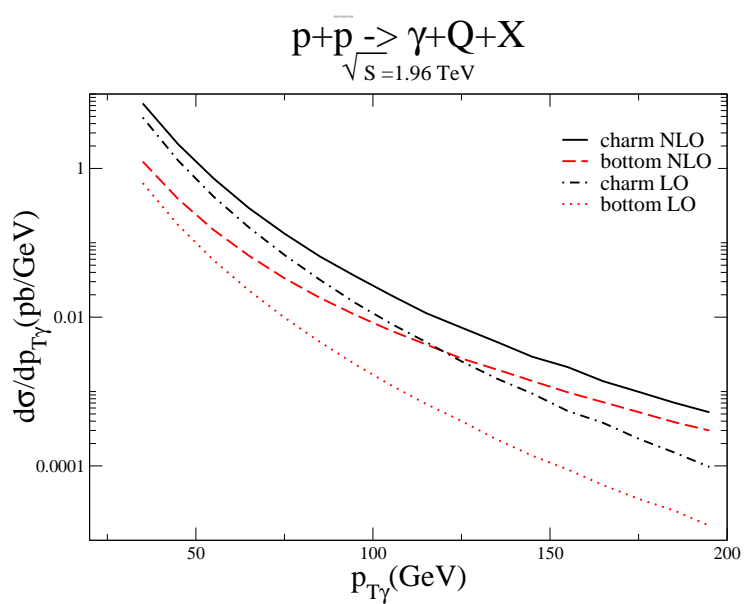

FIG. 7: a comparison between the differential cross sections, $d \sigma / d p_{T \gamma}$ for the production of a direct photon and a bottom quark, and that of a direct photon plus a charm quark at NLO and LO, charm at NLO - solid line and for bottom at NLO- dashed line, charm at LO - dot dashed line, bottom at LO - dotted line

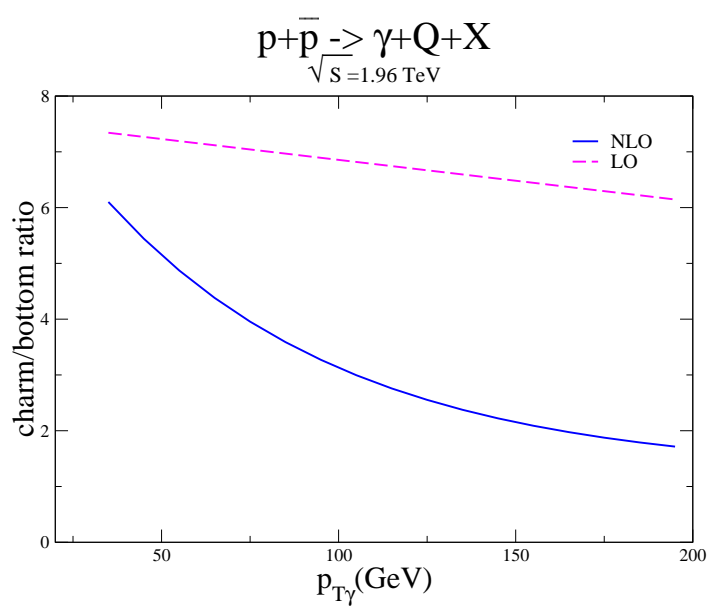

FIG. 8: the ratio of the charm and bottom differential cross sections versus $p_{T \gamma}$, at NLO - solid line and at LO - dashed line

dotted curves (bottom) in Fig,7, and also from Fig, 8 , where the ratio of the NLO and LO charm and bottom differential cross sections is shown. The ratio of the LO cross sections stays almost constant since the main contribution to the LO cross section comes from the Compton subprocess, with the difference between the charm and bottom curves arising from the difference in the charges of the charm and bottom quarks and the relative sizes of the heavy quark PDFs. The ratio of the two LO cross sections depends on the ratio of the charges squared which is $e_{c}^{2} / e_{b}^{2}=4$, and is driven up from that value to about $\sim 7$ because the charm PDF is larger than the bottom PDF.

The fact that the annihilation subprocess dominates the cross section at large $p_{T \gamma}$ also increases the scale dependence of the cross-section in that region. There is no Born term which involves a $q \bar{q}$ initial state and, therefore, the contributions from the annihilation subprocess start in $\mathrm{O}\left(\alpha \alpha_{s}^{2}\right)$. As such, the typical compensation between LO and NLO contributions for this subprocess is missing and the annihilation subprocess can be thought of a leading order. 


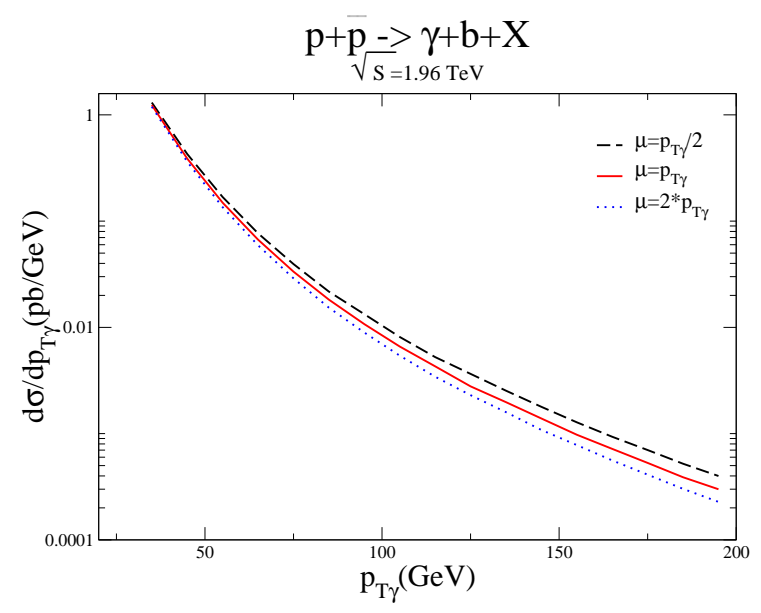

FIG. 9: Scale dependence of the differential cross section, $d \sigma / d p_{T \gamma}$ for the production of a direct photon and a bottom quark, where the three different scales have been set to be equal $\mu=\mu_{r}=$ $\mu_{f}=\mu_{F}, \mu=p_{T \gamma}$ - solid line, $\mu=p_{T \gamma} / 2$ - dashed line, $\mu=2 p_{T \gamma}$ - dotted line

As can be seen from Fig.9 the scale dependence increases at large $p_{T \gamma}$, where the annihilation starts to dominate. The renormalization, $\mu_{r}$, factorization, $\mu_{f}$ and fragmentation, $\mu_{F}$ scales have been set to be equal and are denoted by $\mu$.

\section{B. LHC Predictions}

When data become available from the Large Hadron Collider (LHC), it will be very important to have a good understanding of what Standard Model (SM) processes are going to look like at center of mass energies of $\sqrt{S}=14 \mathrm{TeV}$, as these processes will provide important means of calibrating and understanding the detectors and, ultimately, are likely to provide significant backgrounds to new physics signals. The differential cross section versus the transverse momentum of the photon is shown in Fig.10. It is apparent that the increase of the difference between the NLO (solid line) and the LO (dashed line) grows much less rapidly with increasing $p_{T \gamma}$ than was the case for the Tevatron. In Fig 11 the K-factor, which is the ratio between the NLO and LO cross section for b quarks is shown, which stays stable and is around 2. To understand the difference between the LHC and Tevatron curves, the contributions of the different parts contributing to the LHC cross section are shown in Fig.12. From Fig.12 it can be seen that the annihilation subprocess no longer 


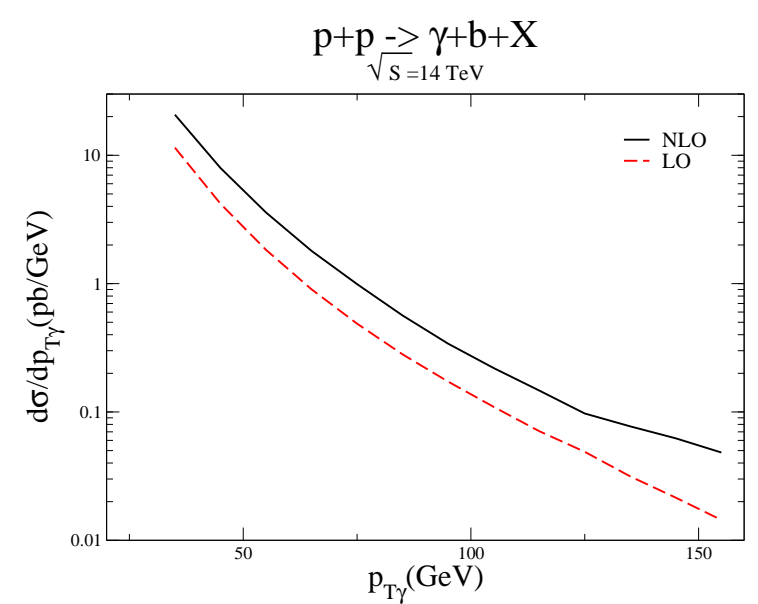

FIG. 10: the differential cross section versus the transverse momentum of the photon $d \sigma / d p_{T \gamma}$ for the production of a direct photon and a bottom quark at LHC center of mass energies, $\sqrt{S}=14 \mathrm{TeV}, \mathrm{NLO}$ - solid line, LO - dashed line

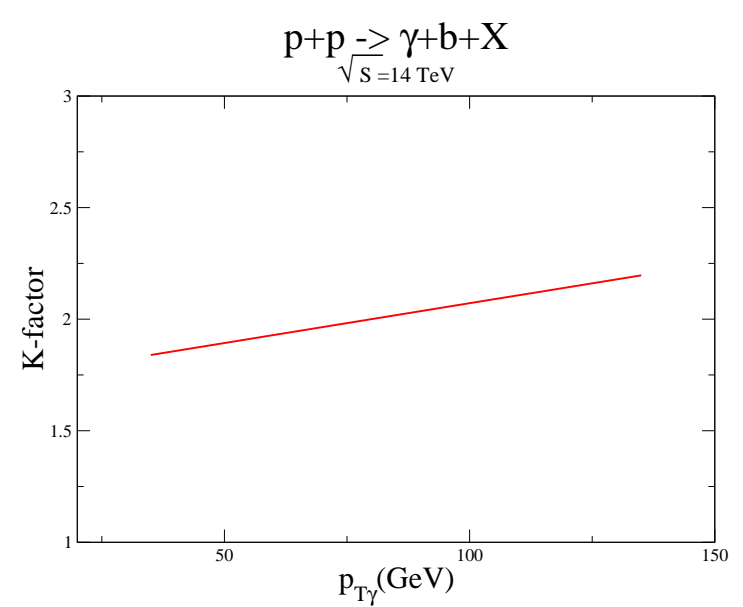

FIG. 11: $\mathrm{K}$ factor, or the ratio of the NLO to the LO differential cross section for $p p \rightarrow b \gamma X$ at $\sqrt{S}=14 \mathrm{TeV}$

drives the cross section at high $p_{T \gamma}$, and now it is the LO, and the $g Q \rightarrow \gamma g Q$ subprocesses that are the most prominent. These differences come about for two reasons. As the LHC collides two beams of protons, instead of the proton and antiproton beams at Fermilab, there is no longer any valence light antiquarks present. Hence, the relative contribution of the annihilation subprocess is decreased. Also, because the LHC will ultimately operate at a center of mass energy which is about seven times larger than that of the Tevatron, lower values of $x \sim p_{T} / \sqrt{s}$ are probed at the LHC. For the kinematic region shown in Fig. 12 the gluon PDF is dominant, accounting for the continued importance of the $g Q$ initiated subprocesses.

An interesting consequence of this pattern of subprocess contributions is that the the dominant parts are all proportional to the heavy quark PDFs. Such was not the case for the Tevatron curves, except for the low end of the $p_{T \gamma}$ range. Accordingly, heavy quark + photon measurements at the LHC will have the potential to provide important cross checks on the perturbatively calculated heavy quark PDFs. These PDFs are likely to provide important contributions to other physics signals - either standard model or new physics - and such 


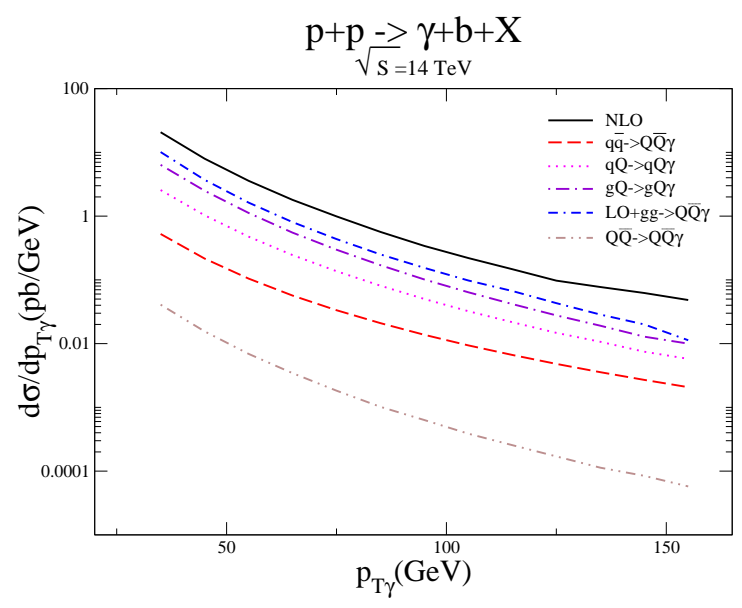

FIG. 12: contributions of the different subprocesses to the differential cross section, $d \sigma / d p_{T \gamma}$ for $p p \rightarrow b \gamma X$ at $\sqrt{S}=14 \mathrm{TeV}, \mathrm{NLO}$-solid line, annihilation $q \bar{q} \rightarrow Q \bar{Q} \gamma$ - dashed line, $q Q \rightarrow q Q \gamma$ - dotted line, $g Q \rightarrow g Q \gamma$ - dot dashed line, $g g \rightarrow Q \bar{Q} \gamma+\mathrm{LO}$ - dash dot dotted line, $Q \bar{Q} \rightarrow \gamma Q \bar{Q}$, and $Q Q \rightarrow \gamma Q Q$ - dot dash dotted line

checks will be an important part of the search for new physics.

\section{NLO Fragmentation and Photon Isolation}

It is interesting to investigate what effect the NLO Fragmentation contributions have upon the cross section. Fig 13 shows the ratio between the full NLO calculation and the cross section with only LO fragmentation. If there are no isolation requirements imposed on the photon, the cross section increases up to $\sim 30 \%$, solid curve in Fig 13 . As mentioned above a photon needs to be isolated in order to give a clear signal at a detector. The isolation requirements affect the photon which is produced by fragmentation the strongest, as it is emitted in close proximity to the parton from which it is fragmenting. This can be seen from the dashed line in Fig. 13, where the NLO fragmentation contribution has now decreased to a few \%. Fig 14 shows the comparison between the differential cross section with the inclusion of isolation and without it. As can be seen this difference is larger at low $p_{T \gamma}$, but the two curves come close to each other with the increase of the photon's transverse momentum, where as seen from Fig. 5 the $q \bar{q} \rightarrow \gamma Q \bar{Q}$ subprocess takes over the cross section. 


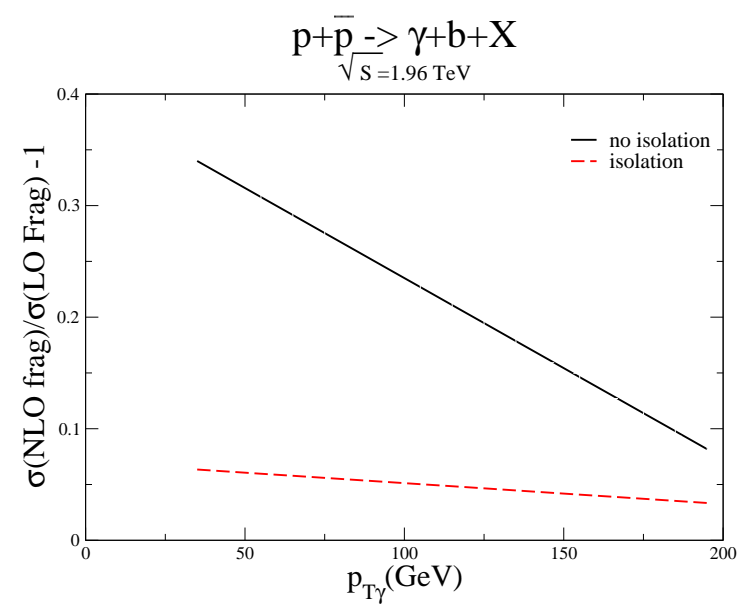

FIG. 13: ratio between the differential cross section $d \sigma / d p_{T \gamma}$, with NLO fragmentation contribution included and the differential cross section with just LO fragmentation included, solid line no isolation required, dashed line -isolation

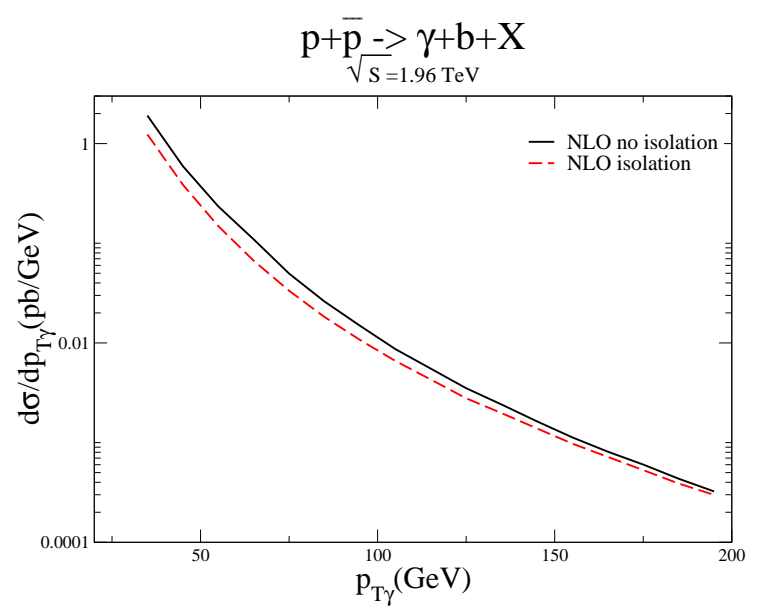

FIG. 14: comparison between the differential cross section, $d \sigma / d p_{T \gamma}$ without isolation requirements and with them, no isolation - solid line , isolation - dashed line

\section{Intrinsic Charm}

In the CTEQ6.6M PDFs used in the previous sections, the charm quark is radiatively generated from the gluon's PDF with the use of the DGLAP equations. Thus it follows that there are no charm quarks present at scales below the charm mass, $m_{c}$, or that the charm PDF, $c(x, \mu)=0$, when $\mu<m_{c}$. This however does not need to be the case, and there are models that study the possibility for an intrinsic charm component of the nucleon [13]. Two such models are the BHPS model, which is a light-cone model, and the sea-like model in which the charm distribution follows the shape of the light flavor sea quarks. The difference between the three cases is shown in Fig 15, where the solid curve shows the CTEQ6.6M or radiatively generated charm scenario, the dashed curve is the CTEQ6.6C2, or BHPS model, and the dotted curve is CTEQ6.6C4 PDF or the sea-like model. The difference between the BHPS distribution and the radiatively generated case are most noticeable at large $\mathrm{x}$, whereas the sea-like model is about equally larger than the CTEQ6.6M PDF at all values of $\mathrm{x}$. How these different PDFs affect the cross section can be seen from Fig,16. The dotted curve shows the cross section generated with the use of the sea-like intrinsic charm PDF, and it 

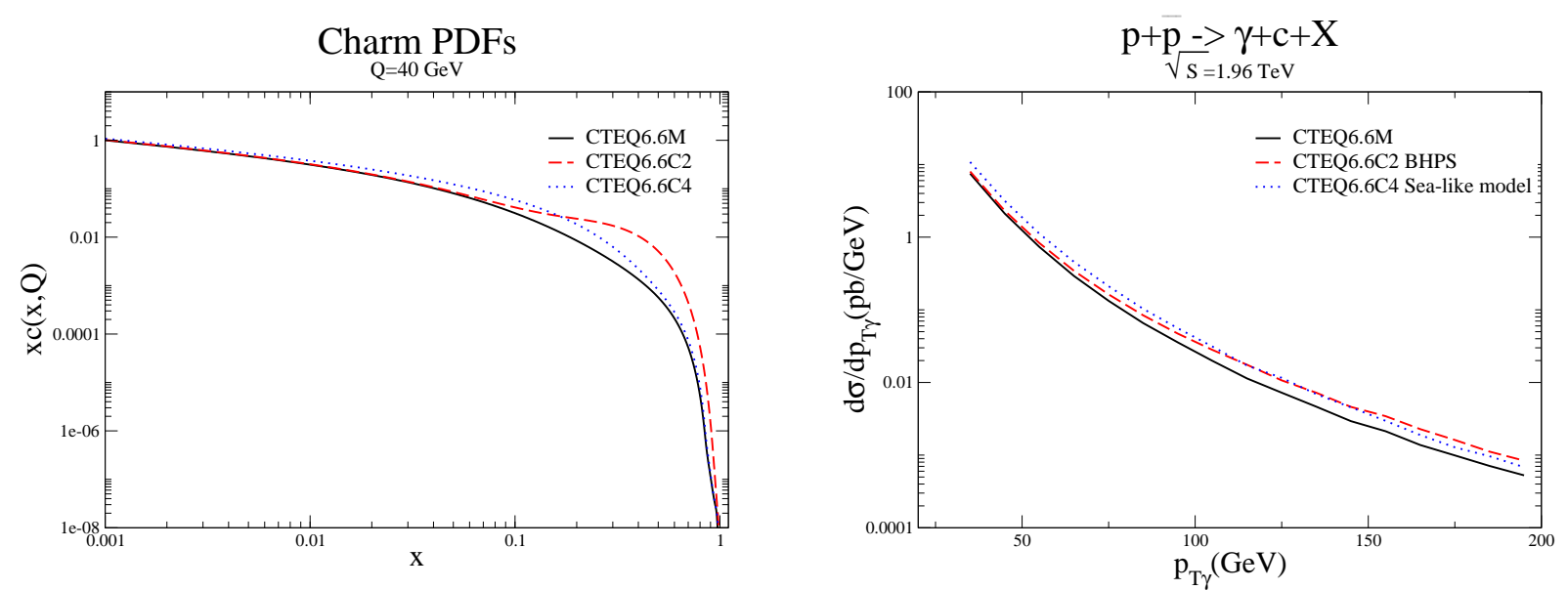

FIG. 15: comparison between the three dif- FIG. 16: the differential cross section, $d \sigma / d p_{T \gamma}$, ferent charm PDFs at scale $Q=40 \mathrm{GeV}$, for the production of a direct photon and a CTEQ6.6M - solid line, CTEQ6.6C2 - dashed charm quark for the three different PDF cases, line, CTEQ6.6C4 - dotted line CTEQ6.6M - solid line, CTEQ6.6C2 - dashed line, CTEQ6.6C4 - dotted line

is larger than the solid curve by about the same amount at all values of $p_{T \gamma}$. The difference between the radiatively generated charm cross section and the BHPS charm however is not great at small transverse momentum, but it increases at large $p_{T \gamma}$, as is expected given the differences between the CTEQ6.6M and CTEQ6.6C2 PDFs at large $\mathrm{x}$.

\section{CONCLUSION}

We have presented the results for the inclusive cross section for the production of a direct photon in association with a heavy quark, $p \bar{p} / p p \rightarrow \gamma Q X$, up to $O\left(\alpha \alpha_{s}^{2}\right)$ with NLO fragmentation included. The inclusion of NLO fragmentation has a noticeable effect on the cross section if no isolation is imposed. However, this effect decreases if isolation cuts, needed for a clean photon signal, are imposed. Predictions were presented for $p \bar{p}$ collisions at $\sqrt{S}=1.96 \mathrm{TeV}$ and for $p p$ collisions at $14 \mathrm{TeV}$. At the Tevatron, due to the $p \bar{p}$ beams, the valence quarks and antiquarks are dominant, and thus it is the annihilation subprocess $q \bar{q} \rightarrow \gamma Q \bar{Q}$ that dominates the cross section at large $p_{T \gamma}$. Therefore the sensitivity to the initial state heavy quarks and their content in the nucleon decreases, and the difference 
between the bottom and charm differential cross sections, $d \sigma / d p_{T \gamma}$, also diminishes. At the LHC, where two beams of protons are colliding, there are no longer any valence antiquarks present, and processes with initial gluons and heavy quarks dominate. Thus there should be a greater possibility to learn more about the heavy quark role in the nucleon. In particular, the perturbatively calculated heavy quark PDFs may be checked using such data.

[1] J.F. Owens, Rev. Mod. Phys. 59,465 (1987).

[2] P. Aurenche et al., Phys. Rev. D 39, 3275 (1989).

[3] J. Huston et al., Phys. Rev. D 51, 6139 (1995).

[4] P. Aurenche et al., Eur. Phys. J. C 9, 107 (1999).

[5] P. Aurenche et al., Phys. Rev. D 73, 094007 (2006).

[6] D0 Collaboration: V. Abazov, et al,. arXiv:0804.1107v2 [hep-ex], 2008.

[7] B. Bailey, E.L. Berger, and L.E. Gordon, Phys.Rev. D 54, 1896 (1996).

[8] E.L. Berger and L.E. Gordon, Phys.Rev. D 54, 2279 (1996).

[9] M. Stratmann and W. Vogelsang, Phys.Rev. D 52, 1535 (1995).

[10] E.L. Berger and L.E. Gordon Phys.Rev. D 58, 114024 (1998).

[11] B.W. Harris and J.F. Owens, Phys.Rev. D 65, 094032 (2002).

[12] P.M. Nadolsky, Phys.Rev. D 78, 013004 (2008).

[13] J. Pumplin, H.L. Lai, and W.K. Tung, Phys.Rev. D 75, 054029 (2007). 